\title{
Ivermectin inhibits the sporogony of Plasmodium falciparum in Anopheles gambiae
}

Kevin C Kobylinski ${ }^{*}$, Brian D Foy ${ }^{2}$ and Jason H Richardson ${ }^{1}$

\begin{abstract}
Background: When ingested in a blood meal, ivermectin has been shown to reduce the survivorship of Anopheles gambiae in the laboratory and field. Furthermore, ivermectin mass drug administrations in Senegal have been shown to reduce the proportion of Plasmodium falciparum-sporozoite-containing An. gambiae. This study addresses whether ivermectin inhibits sporogony of $P$. falciparum in An. gambiae.

Methods: Anophele gambiae s.s. G3 strain were fed two concentrations of ivermectin ( $\mathrm{LC}_{25}$ and $\left.\mathrm{LC}_{5}\right)$ along with P. falciparum NF54 in human blood meals at staggered intervals. Mosquitoes ingested ivermectin concurrent with parasites (DPI 0), or at three (DPI 3), six (DPI 6), and nine (DPI 9) days post parasite ingestion, or three days prior (DPI -3) to parasite ingestion. Mosquitoes were dissected at seven, twelve or fourteen days post parasite ingestion and either oocyst or sporozoite prevalence was recorded. To determine if $P$. falciparum sporozoite-containing An. gambiae were more susceptible to ivermectin than uninfected controls, survivorship was recorded for mosquitoes which ingested $P$. falciparum or control blood meal on DPI 0 and then a second blood meal containing ivermectin $\left(\mathrm{LC}_{25}\right)$ on DPI 14.
\end{abstract}

Results: Ivermectin $\left(\mathrm{LC}_{25}\right)$ co-ingested (DPI 0) with parasites reduced the proportion of An. gambiae that developed oocysts $\left(X^{2}=15.4842, P=0.0002\right)$ and sporozoites $\left(X^{2}=19.9643, P<0.0001\right)$. Ivermectin $\left(L C_{25}\right)$ ingested DPI 6 $\left(X^{2}=8.5103, P=0.0044\right)$ and $9\left(X^{2}=14.7998, P<0.0001\right)$ reduced the proportion of $A n$. gambiae that developed sporozoites but not when ingested DPI $3\left(x^{2}=0.0113, P=1\right)$. Ivermectin $\left(L C_{5}\right)$ co-ingested (DPI 0$)$ with parasites did not reduce the proportion of An. gambiae that developed oocysts $\left(X^{2}=4.2518, P=0.0577\right)$ or sporozoites $\left(X^{2}=2.3636, P=0.1540\right)$, however, when ingested DPI -3 the proportion of An. gambiae that developed sporozoites was reduced $\left(X^{2}=8.4806, P=0.0047\right)$. Plasmodium falciparum infection significantly reduced the survivorship of An. gambiae that ingested ivermectin $\left(\mathrm{LC}_{25}\right)$ on DPI 14 compared to control mosquitoes that ingested a primary blood meal without parasites $\left(X^{2}=4.97, P=0.0257\right)$.

Conclusions: Ivermectin at sub-lethal concentrations inhibits the sporogony of $P$. falciparum in An. gambiae. These findings support the utility of ivermectin for $P$. falciparum transmission control.

Keywords: Anopheles gambiae, Plasmodium falciparum, Ivermectin, Transmission

\section{Background}

Novel Anopheles vector control methods are needed for current malaria elimination and eradication efforts [1]. Ivermectin mass drug administration (MDA) to humans has been suggested as a possible vector control method to reduce Plasmodium transmission [2-8]. Ivermectin MDA to humans meets several of the criteria outlined by the Malaria Eradication Research

\footnotetext{
*Correspondence: kevin.c.kobylinski.ctr@us.army.mil

${ }^{1}$ Entomology Branch, Walter Reed Army Institute of Research, 503 Robert Grant Ave, Silver Spring, MD 20910, USA

Full list of author information is available at the end of the article
}

Agenda Consultative Group on Vector Control for novel vector control interventions, including: the ability to target exophagic and exophilic vectors, integration with current vector control efforts [5,6], novel mode of action $[9,10]$ from the currently used insecticides [11], avoids known mosquito behavioural and physiological resistance mechanisms, and can affect the vector population age structure [7].

The vectorial capacity equation provides a framework that defines the factors that regulate Plasmodium transmission by Anopheles vectors [12]. The current vector 
control strategies of indoor residual spraying with insecticides and long lasting insecticide treated nets primarily affect the daily probability of mosquito survivorship and/or reduce vector-host contact [13,14]. However, there are several other variables in the vectorial capacity equation that vector control methods could impact which would reduce Plasmodium transmission.

The most influential variable of vectorial capacity is the daily probability of mosquito survivorship $[12,15]$. In vitro laboratory studies have demonstrated that ivermectin can reduce the daily probability of survivorship of numerous Anopheles Plasmodium-vectors, including Anopheles gambiae s.s. at physiologically relevant concentrations found in humans after ingestion of ivermectin $[4,16,17]$. The second most influential variable of the vectorial capacity equation is the daily probability of a mosquito feeding on a human, which is a factor of the mosquito host preference index and the feeding frequency. Anopheles gambiae s.s. blood-feeds almost exclusively on humans with a median host preference index of 0.939 [18]. The feeding frequency of An. gambiae s.s. was significantly delayed when ivermectin was ingested in a blood meal at mosquito sub-lethal concentrations $[4,19]$. The vector density relation to the host is another variable of vectorial capacity that may be affected by ivermectin MDA. Ivermectin not only reduces the survivorship of An. gambiae s.s., but it also causes a knockdown effect and a recovery delay post blood feeding [19] which likely further reduces mosquito populations due to desiccation, starvation, and predation. Ivermectin has also been shown to reduce An. gambiae fecundity [16]. This laboratory evidence and modelling data [7] suggests that vector density in relation to the host may be temporarily reduced after ivermectin MDA to humans, but this remains to be demonstrated in a field setting.

Ivermectin MDAs to humans are performed annually in many parts of sub-Saharan Africa for Onchocerca volvulus transmission suppression by the African Program for Onchocerciasis Control (APOC) and Wuchereria bancrofti transmission suppression by the Global Programme to Eliminate Lymphatic Filariasis [20]. In south-eastern Senegal, indoor-resting blood-fed An. gambiae s.l. were collected before and after routine APOC ivermectin MDAs from three pairs of treated and control villages and this work clearly demonstrated that An. gambiae s.s. survivorship was significantly reduced for approximately one week following ivermectin MDA [5]. Further analysis of fieldcollected mosquitoes revealed that ivermectin MDA also reduced the proportion of Plasmodium falciparum sporozoite-containing (i.e. infectious) An. gambiae s.s. for at least two weeks post-MDA [6].

There are several possible explanations for this reduction in the proportion of P. falciparum-infectious $A n$. gambiae s.s, which are not exclusive of each other. Modelling has predicted that the An. gambiae population age structure following ivermectin MDA temporarily shifts to younger age classes, therefore, fewer P. falciparuminfectious mosquitoes are present for several weeks [7]. Field work is currently being performed in Senegal to gather empirical evidence as to whether this population age structure shift occurs. It may also be that $P$. falciparum-infectious mosquitoes are more susceptible than non-infected mosquitoes to ivermectin and thus are preferentially removed from the population of mosquitoes. Finally, ivermectin may be sporontocidal and thus lower the proportion or prevalence of Anopheles ingesting an infective Plasmodium blood meal that successfully become infectious or lengthen the extrinsic incubation period of Plasmodium in the Anopheles vector. These latter three possibilities were explored below in laboratory experiments with P. falciparum and An. gambiae s.s.

\section{Methods \\ Parasites}

Gametocyte cultures of P. falciparum (NF54) line were cultured in vitro following the modified Trager and Jensen method $[21,22]$ in human erythrocytes group $\mathrm{O}+$ (Key Biologics LLC, Memphis, TN) and RPMI 1640 medium (Life Technologies, Grand Island, NY) supplemented with $25 \mathrm{mM}$ HEPES, $25 \mathrm{mM} \mathrm{NaHCO}, 50 \mathrm{mg} / \mathrm{L}$ hypoxanthine (Sigma-Aldrich, St. Louis, MO) and 10\% (v/v) type A+ human serum (Key Biologics LLC, Memphis, TN). Media was changed daily and parasites were blood fed to mosquitoes 15 days post subculture. For mosquito blood meals cultures were transferred from flasks to $50 \mathrm{ml}$ conical tubes and centrifuged at 1600 RPMs for ten minutes at $37^{\circ} \mathrm{C}$ and culture media supernatant was removed. Red blood cells (RBCs) and human serum were mixed in a 1:1 ratio and fed to mosquitoes via glass membrane feeders sealed with parafilm at $37^{\circ} \mathrm{C}$.

\section{Mosquitoes}

Anopheles gambiae s.s. (G3 strain) were obtained from the National Institutes of Health, raised at $26-27^{\circ} \mathrm{C}, 80 \%$ relative humidity, and a 12:12 light:dark cycle. Larvae were raised on a diet of ground Tetramin ${ }^{\circledR}$ fish food. Adults were provided $10 \%$ sucrose solution ad libitum. Adult mosquitoes were between four and seven days post emergence when they were fed a $P$. falciparuminfectious blood meal.

\section{$\mathrm{LC}_{50}$ calculations}

The powdered ivermectin formulation was obtained from Sigma-Aldrich (St. Louis, MO). Ivermectin was diluted in dimethyl sulfoxide (DMSO) to $10 \mathrm{mg} / \mathrm{ml}$ and aliquots were frozen at $-20^{\circ} \mathrm{C}$. Frozen aliquots of ivermectin were thawed and serially diluted in phosphate 
buffered saline (PBS) prior to addition to blood meals consisting of packed RBCs and human serum mixed 1:1 heated to $37^{\circ} \mathrm{C}$ prior to mixing. Ten $\mu \mathrm{l}$ of varied concentrations of ivermectin in PBS were added to $990 \mu \mathrm{l}$ of blood meal to reach the final concentrations offered to mosquitoes. Multiple concentrations of ivermectin were fed to adult An. gambiae s.s. between four and seven days post emergence to determine the lethal concentration that killed $50 \%, 35 \%, 25 \%$, and $5 \%$ of the mosquitoes ( $\mathrm{LC}_{50}, \mathrm{LC}_{35}, \mathrm{LC}_{25}$, and $\mathrm{LC}_{5}$ ). After blood feeding, 50 fully engorged mosquitoes were gently transferred by aspiration to $0.5 \mathrm{~L}$ cardboard cartons and held in a different incubator at $26^{\circ} \mathrm{C}, 80 \%$ relative humidity, and a $12: 12$ light:dark cycle. Engorged mosquitoes were held for seven days with access to $10 \%$ sucrose solution and survivorship was monitored every 24 hours and dead mosquitoes were removed from the containers daily. Six replicates were performed.

Effect of ivermectin on Plasmodium falciparum sporogony Anopheles gambiae s.s. were fed cultured gametocytaemic RBCs with various concentrations of ivermectin, $\mathrm{LC}_{25}$ and $\mathrm{LC}_{5}$, and at various time points in relation to the infectious blood meals. Ivermectin- $\left(\mathrm{LC}_{25}\right)$-containing blood meals were administered concomitantly with parasites (DPI 0), or three (DPI 3), six (DPI 6), and nine (DPI 9) days after (post) the infectious (DPI) blood meal. Ivermectin-( $\left(\mathrm{LC}_{5}\right)$-containing blood meals were administered concomitantly with parasites (DPI 0 ), or three (DPI -3) days before parasites. Control mosquitoes were fed in a separate container with the same blood meal schedule, only ivermectin was replaced with DMSO at equivalent concentrations in PBS. After blood feeding, fully engorged mosquitoes were gently transferred by aspiration to new $3.8 \mathrm{~L}$ cardboard cartons and held in a different incubator at $26^{\circ} \mathrm{C}, 80 \%$ relative humidity, and a 12:12 light:dark cycle. Mosquitoes were held for fourteen days after the infectious blood feed with $10 \%$ sucrose solution ad libitum. Mosquitoes fed ivermectin $\left(\mathrm{LC}_{25}\right.$ and $\left.\mathrm{LC}_{5}\right)$ concomitantly with infectious gametocytes (DPI 0 ) were dissected on day post infection (DDPI) 7 and oocysts were enumerated. Midguts were dissected with forceps into saline on a microscope slide and stained for 15 minutes in methylene blue $(0.1 \%)$ (Fisher Scientific, Fair Lawn, NJ), pressed between a cover slip and viewed at 200X magnification with a MT5000 Series biological microscope (Meiji Techno, Santa Clara, CA). A delay in blood meal digestion caused by ivermectin [4] ingested on DPIs 3 and 6 obscured the ability to view oocysts on the midgut, therefore, prevalence and intensity were not determined at those time points. On DDPI 12 and 14, salivary glands were dissected with forceps into saline on a microscope slide, pressed between a cover slip, and viewed at 200X magnification with a MT5000 Series biological microscope. Prevalence of sporozoites in salivary glands were recorded for each mosquito. Four replicates of ivermectin $\left(\mathrm{LC}_{5}\right.$ or $\left.\mathrm{LC}_{25}\right)$ concentration and DPI $(-3,0,3,6$, or 9) combinations were performed and approximately twenty mosquitoes were dissected for each control and treatment group on each DDPI. Two additional replicates of ivermectin $\left(\mathrm{LC}_{25}\right)$ DPI 0 were performed in order to obtain enough visible midguts with oocyst infections for statistical analyses.

\section{Effect of Plasmodium falciparum infection on mosquito survivorship post ivermectin ingestion}

Anopheles gambiae s.s. were fed either gametocytaemic or non-gametocytaemic control blood meals and held in the insectary for fourteen days in $3.8 \mathrm{~L}$ cartons. On DPI 14 , ten mosquitoes were dissected as described above to confirm at least $90 \%$ P. falciparum sporozoite presence in salivary glands. If the mosquitoes met the infection criteria status, then both infected and control cartons were fed a secondary ivermectin $\left(\mathrm{LC}_{25}\right)$-containing blood meal. Approximately 50 mosquitoes were transferred by aspiration to $0.5 \mathrm{~L}$ cartons and held as described above. Mosquito survivorship was monitored every 24 hours and dead mosquitoes were removed from the containers daily until all mosquitoes were dead. Five replicates were performed.

\section{Statistical analysis}

A non-linear mixed model with probit analysis was used to calculate $\mathrm{LC}_{50}, \mathrm{LC}_{35}, \mathrm{LC}_{25}$, and $\mathrm{LC}_{5}$ values as described previously [4]. The proportion of mosquitoes with oocysts or sporozoites was compared by Fisher's Exact test. In order to determine if ivermectin lengthened the extrinsic incubation period the rates of change in the proportion of sporozoite positive mosquitoes between DDPI 12 and DDPI 14 were analyzed by logistic regression (SAS Proc GLIMMIX) [23] with effects for treatment (ivermectin, control), period (DDPI 12, DDPI 14 ), and treatment by period with replicate as a random effect. The treatment by period interaction tests whether the change in the proportion of sporozoite positive mosquitoes between DDPI 12 and DDPI 14 differs between ivermectin and control treatments. These above statistical analyses were performed with Statistical Analysis Software (SAS Institute Inc., Cary, NC). Oocyst intensity was compared by the Mann-Whitney $U$ test. To determine if $P$. falciparum infection altered mosquito survivorship post-ivermectin ingestion survival analysis data were analysed by the Mantel-Cox method. Mann-Whitney U tests and Mantel-Cox tests were performed with PRISM 5 (GraphPad Software, Inc., San Diego, CA). 


\section{Results}

$\mathrm{LC}_{50}$ calculations

The lethal concentrations of ivermectin fed to An. gambiae s.s. were calculated as: $\mathrm{LC}_{50}=15.9 \mathrm{ng} / \mathrm{ml} 95 \%$ C.I. $[14.6,17.3], \mathrm{LC}_{35}=12.7 \mathrm{ng} / \mathrm{ml}[11.3,13.9], \mathrm{LC}_{25}=10.7$ $\mathrm{ng} / \mathrm{ml}[9.3,12.0], \mathrm{LC}_{5}=6.1 \mathrm{ng} / \mathrm{ml}[4.8,7.4](\mathrm{n}=3355)$.

\section{Effect of ivermectin on Plasmodium falciparum sporogony} When ivermectin $\left(\mathrm{LC}_{25}\right)$ was co-ingested with $P$. falciparum (ie. DPI 0) it reduced the prevalence or proportion of An. gambiae that developed oocysts at DDPI $7\left(\chi^{2}=\right.$ 15.4842, $P=0.0002, \mathrm{n}=89)$ and sporozoites at DDPI 12 $\left(\chi^{2}=13.4741, P=0.0003, \mathrm{n}=97\right)$ and at DDPI $14\left(\chi^{2}=\right.$ $19.9643, P<0.0001, \mathrm{n}=136$ ) (Figure 1$)$. The oocyst intensity per successfully infected mosquito fed ivermectin $\left(\mathrm{LC}_{25}\right)$ (mean $=21.0 \pm 2.93$ s.e.) at DPI 0 was not reduced relative to controls (mean $=21.5 \pm 2.94$ s.e. $)(P=$ $0.8139, \mathrm{n}=136)$. When ivermectin $\left(\mathrm{LC}_{25}\right)$ was ingested on DPI 3, it did not reduce the proportion of An. gambiae that contained sporozoites at DDPI $12\left(\chi^{2}=4.3718, P=\right.$ $0.0556, \mathrm{n}=112)$ or at DDPI $14\left(\chi^{2}=0.0113, P=1, \mathrm{n}=\right.$ 145) (Figure 2). However, ivermectin $\left(\mathrm{LC}_{25}\right)$ ingested on DPI 6 and DPI 9 reduced the proportion of An. gambiae that contained sporozoites at DDPI 12 and DDPI 14 [DPI 6 at DDPI $12\left(\chi^{2}=12.4919, P=0.0006, \mathrm{n}=95\right)$ and DPI 6 at DDPI $14\left(\chi^{2}=8.5103, P=0.0044, \mathrm{n}=135\right)$; DPI 9 at DDPI $12\left(\chi^{2}=16.2056, P<0.0001, \mathrm{n}=132\right)$ and DPI 9 at DDPI $14\left(\chi^{2}=14.7998, P<0.0001, \mathrm{n}=161\right)$ ] (Figure 2).

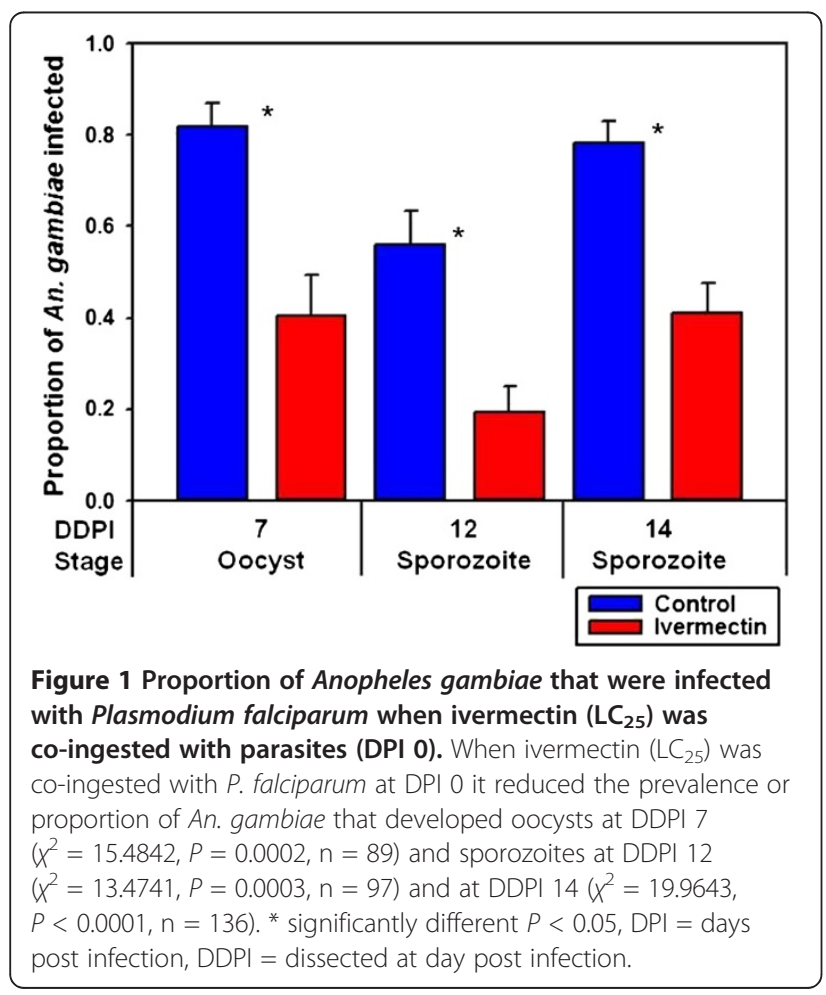

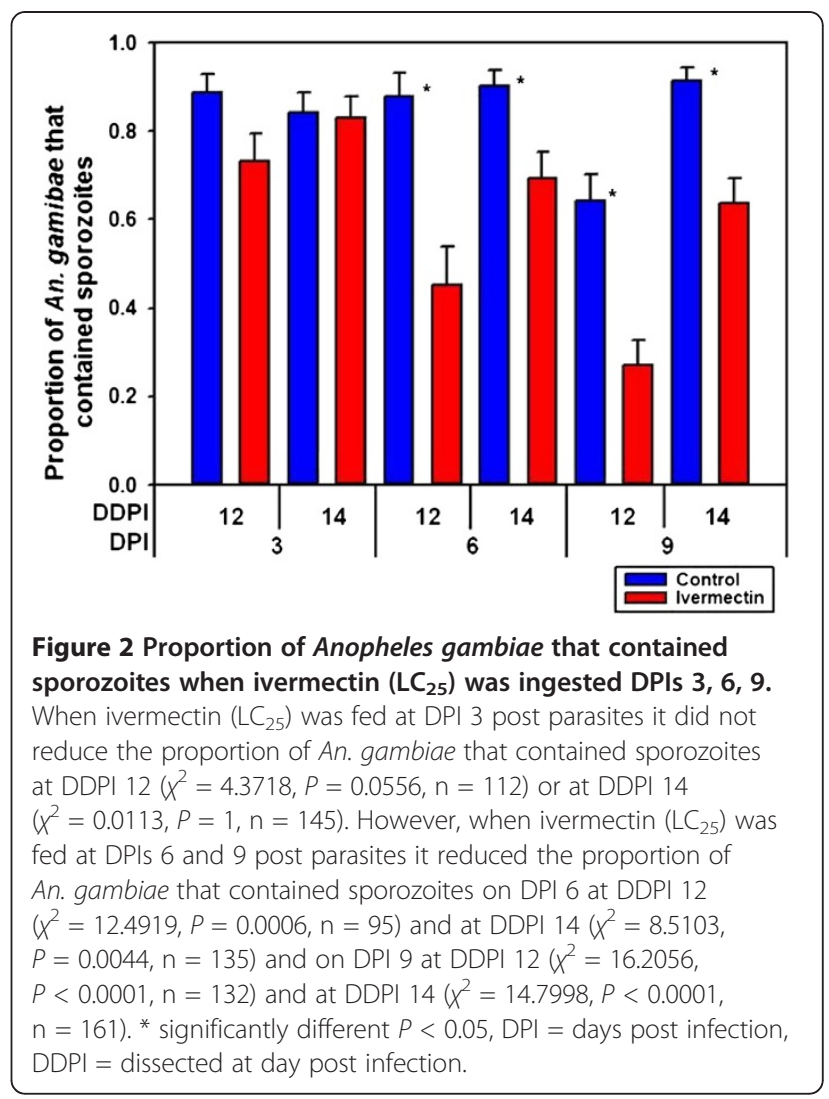

When ivermectin $\left(\mathrm{LC}_{5}\right)$ was co-ingested with $P$. falciparum (DPI 0), it did not reduce the prevalence or proportion of An. gambiae that developed oocysts at DDPI $7\left(\chi^{2}=\right.$ $4.2518, P=0.0577, \mathrm{n}=115)$ or sporozoites at DDPI 12 $\left(\chi^{2}=0.5937, P=0.5101, \mathrm{n}=149\right)$ or at DDPI $14\left(\chi^{2}=\right.$ $2.3636, P=0.1540, \mathrm{n}=156$ ) (Figure 3 ). The oocyst intensity per successfully infected mosquito fed ivermectin $\left(\mathrm{LC}_{5}\right)$ on DPI 0 (mean $=15.4 \pm 2.53$ s.e.) was not reduced compared to controls $($ mean $=13.1 \pm 2.34$ s.e. $)(P=0.9278, \mathrm{n}=84)$. However, when ivermectin $\left(\mathrm{LC}_{5}\right)$ was ingested three days before a $P$. falciparum blood meal (DPI -3 ) it reduced the proportion of $A n$. gambiae that contained sporozoites at DDPI $12\left(\chi^{2}=4.6333, P=0.0406, \mathrm{n}=95\right)$ and at DDPI 14 $\left(\chi^{2}=8.4806, P=0.0047, \mathrm{n}=155\right)$ (Figure 4$)$.

Ivermectin $\left(\mathrm{LC}_{25}\right)$ did not delay the extrinsic incubation period of $P$. falciparum when ingested on DPI 0 (F-value $=0.01, P=0.9308, \mathrm{n}=233), \mathrm{DPI} 3($ F-value $=$ 1.89, $P=0.2116, \mathrm{n}=257)$, DPI $6($ F-value $=0.27, P=$ $0.6182, \mathrm{n}=230$ ), or DPI 9 (F-value $=0.08, P=0.7796$, $\mathrm{n}=295)$ nor did ivermectin $\left(\mathrm{LC}_{5}\right)$ delay sporogony when ingested at DPI 0 (F-value $=0.59, P=0.4622, \mathrm{n}=304)$ or DPI $-3($ F-value $=0.01, P=0.9238, \mathrm{n}=250)$.

\section{Effect of Plasmodium falciparum infection on mosquito} survivorship post ivermectin ingestion

Plasmodium falciparum infection significantly reduced the survivorship of An. gambiae that ingested ivermectin 


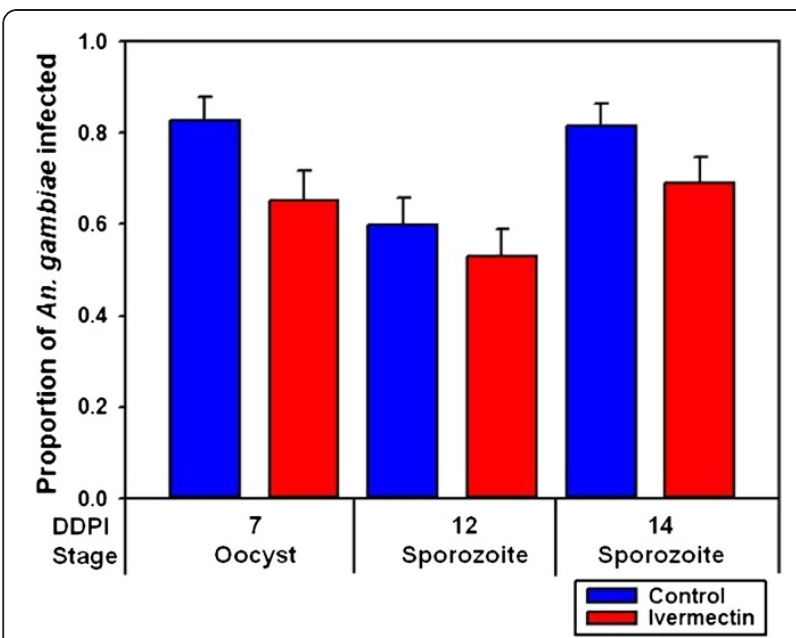

Figure 3 Proportion of Anopheles gambiae that were infected with Plasmodium falciparum when ivermectin $\left(\mathrm{LC}_{5}\right)$ was co-ingested with parasites (DPI 0). When ivermectin ( $L C_{5}$ ) was co-ingested with $P$. falciparum on DPI 0 it did not reduce the proportion of An. gambiae that developed oocysts at DDPI 7 $\left(x^{2}=4.2518, P=0.0577, n=115\right)$ or sporozoites at DDPI 12 $\left(x^{2}=0.5937, P=0.5101, \mathrm{n}=149\right)$ or at DDPI $14\left(x^{2}=2.3636\right.$, $P=0.1540, \mathrm{n}=156)$

$\left(\mathrm{LC}_{25}\right)$ on DPI 14 compared to control mosquitoes that received a primary blood meal without $P$. falciparum $\left(\chi^{2}=\right.$ 4.97, $P=0.0257, \mathrm{n}=450$ ) (Figure 5). Preliminary data did not indicate that $P$. falciparum reduced the survivorship of $A n$. gambiae that co-ingested ivermectin $\left(\mathrm{LC}_{35}\right)$ on DPI 0 compared to control mosquitoes that received only a blood meal $\left(\chi^{2}=0.1054, P=0.7454, \mathrm{n}=61\right)$, therefore, further replicates were not performed. Preliminary data indicated that $P$. falciparum infection compared to a

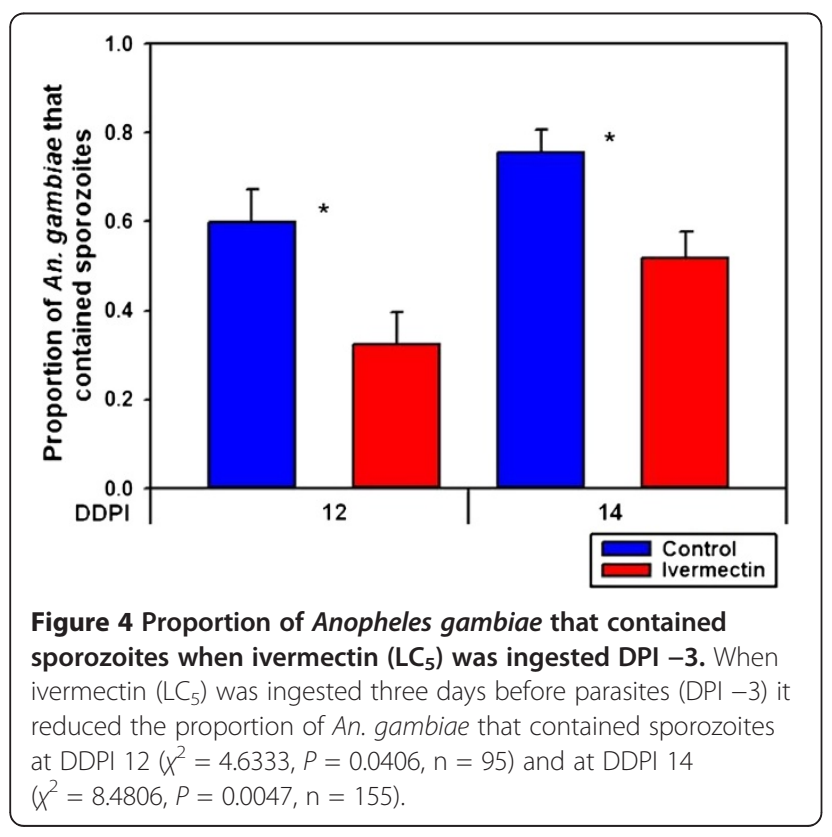

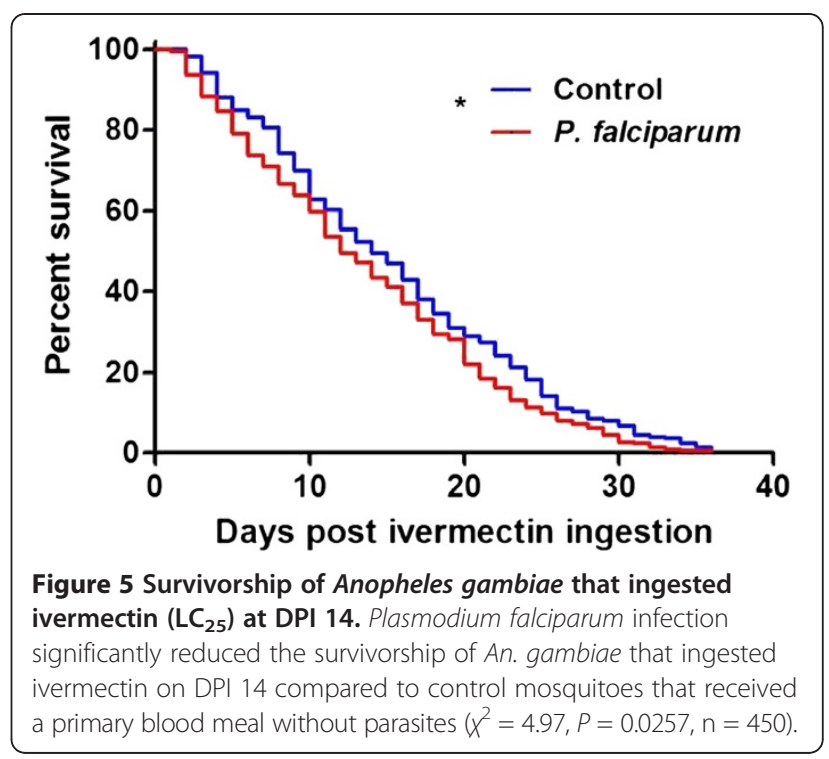

control blood meal on DPI 0 did not reduce $A n$. gambiae survivorship $\left(\chi^{2}=0.3518, P=0.5531, \mathrm{n}=59\right)$.

\section{Discussion}

This study demonstrates that, in several different combinations, ivermectin is sporontocidal and reduces the prevalence or proportion of $A n$. gambiae ingesting an infective $P$. falciparum blood meal that become sporozoite positive. Plasmodium falciparum-infectious An. gambiae may be more susceptible to ivermectin compared to non-infected mosquitoes. Ivermectin does not appear to lengthen the extrinsic incubation period of $P$. falciparum in An. gambiae.

Ivermectin $\left(\mathrm{LC}_{25}\right)$ ingested concomitantly with parasites (DPI 0) had the most dramatic effect on P. falciparum sporogony of any ivermectin concentration or DPI combination (Figures 1-4). While the proportion of mosquitoes that developed oocysts were reduced by ivermectin treatment, the number of oocysts per infected mosquito was not reduced, which implies that ivermectin exerts an 'all-or-nothing' effect on oocyst establishment and development. There was a significant reduction in parasite development when ivermectin $\left(\mathrm{LC}_{25}\right)$ was ingested at DPI 6 and DPI 9 but not at DPI 3 (Figure 2). This suggests that ivermectin can affect latestage but not early-stage oocyst development. Finally, ivermectin $\left(\mathrm{LC}_{5}\right)$ ingested on DPI 0 had no effect on oocyst or sporozoite prevalence (Figure 3), however when ivermectin $\left(\mathrm{LC}_{5}\right)$ was ingested prior to parasites (DPI -3) there was a significant reduction in sporozoite prevalence (Figure 4). These results suggest that ivermectin has a modulating effect on the mosquito host environment that is unfavourable for sporogonic development. 
While this report does not attempt to elucidate the mode of action of ivermectin on $P$. falciparum sporogony, the data presented may offer some insight. Ivermectin is a lipophilic molecule that embeds itself in membranes and interacts with transmembrane portions of ion channels [24]. In invertebrates, ivermectin interacts with glutamate-gated chloride ion channels in neuronal and neuromuscular tissues, which leads to flaccid paralysis $[9,10,25]$. There may also be some interaction of ivermectin with $\gamma$-aminobutyric acid-gated chloride channels [26-28]. Neither of these chloride ion channels are present in $P$. falciparum and ivermectin does not have relevant activity against blood-stage $P$. falciparum at concentrations found in humans post oral treatment. Growth of blood-stage P. falciparum (K1 isolate) was inhibited by ivermectin but the mean $\mathrm{IC}_{50}$ and $\mathrm{IC}_{90}$ values were 8.0 and $35.0 \mu \mathrm{g} / \mathrm{ml}$ [29] which are several hundred to thousand times higher than concentrations used in the current study (6.1 and $10.7 \mathrm{ng} / \mathrm{ml}$ ) and normal levels found in human venous plasma after ingesting ivermectin $(150 \mu \mathrm{g} / \mathrm{kg})$ [30]. Indeed, oral ivermectin $(200 \mu \mathrm{g} / \mathrm{kg})$ treatment of P. falciparum-infected humans had no effect on blood-stage parasites [31]. Similarly, no relevant effects of ivermectin were demonstrated against the Apicomplexa coccidian Eimeria muris or dozens of species of bacteria and fungi [32]. Nevertheless, it may be possible that unknown ivermectin targets specific to sporogonic $P$. falciparum stages exist which may explain the activity against late-stage but not early-stage oocysts (Figure 2). The broad activity of ivermectin is evident from recent in vitro evidence that demonstrates that ivermectin can effect viral replication of human immunodeficiency virus and several arthropod-transmitted Flaviviruses by targeting virus NS3 helicase activity [33] or inhibiting nuclear import [34].

The observed 'all-or-nothing' effect of ivermectin on oocyst development in this report is more congruent with a sporontocidal mechanism whereby ivermectin acts on the mosquito rather than the parasite. Ivermectin may alter some aspect of the mosquito midgut physiology that impacts parasite development or the drug may stimulate enhanced anti-Plasmodium innate immunity. Regarding the latter possibility, no melanized oocysts from dissected midguts were observed from any control or ivermectin-treated mosquitoes with any ivermectin concentration or DPI combination. The data showing that ivermectin $\left(\mathrm{LC}_{5}\right)$ only inhibits sporogony when ingested DPI -3 , but not at DPI 0 , suggests that this treatment may alter some aspect of the midgut physiology that prevents parasite establishment, such as mosquito peritrophic matrix formation or blood meal digestion prior to parasite ingestion. Previous work has shown ivermectin to delay the formation and reduce the thickness of the peritrophic matrix in Aedes aegypti [35].
Delays in blood meal digestion by $A n$. gambiae that had ingested an ivermectin-containing blood meal have been reported previously [4] and were observed in these experiments as well. There is a complex interaction of the $P$. falciparum ookinete with the peritrophic matrix [36,37] and the midgut epithelium [38], which may be affected by ivermectin.

Previous investigators have found accelerated development kinetics of wild strains of $P$. falciparum in wild Anopheles in that most ookinetes were formed in the blood meal before twenty-four hours [39], while with the in vitro NF54 strain in colonized Anopheles the ookinetes were formed in the blood meal after twenty-four hours [40-42]. The ivermectin studies presented here should be replicated with wild $P$. falciparum isolates and various An. gambiae strains and other Anopheles species to ensure that the effects of ivermectin observed with NF54 represent what occurs in the field. The effects of ivermectin on other Plasmodium species in various Anopheles species should be investigated as well.

Ivermectin did not appear to lengthen the extrinsic incubation period $(n)$, for any ivermectin concentration or DPI combination. More frequent dissections and sporozoite observation on DDPIs 10, 11, 13 and increased replication would provide more data for the general linear mixed models which may be better than the inputs used for this study. Extending dissections beyond DDPI 14 may not provide relevant information as the proportion of infected control mosquitoes at DDPI 7 matched the proportion of infectious control mosquitoes at DDPI $14\left(\chi^{2}=0.2803, P=0.6717, \mathrm{n}=135\right)$ and the proportion of infected ivermectin-treated mosquitoes at DDPI 7 matched the proportion of infectious ivermectin-treated mosquitoes at DDPI $14\left(\chi^{2}=0.0, P=1, \mathrm{n}=90\right)$ (Figure 1). Alternative methods such as enumerating ruptured oocysts at time points beyond DDPI 7 may be necessary to determine if the extrinsic incubation is altered.

A significant, although modest, reduction in $P$. falciparum-infectious An. gambiae survivorship compared to uninfected counterparts was demonstrated $\left(\chi^{2}=4.97\right.$, $P=0.0257$ ) (Figure 5). As the effect of $P$. falciparum on Anopheles survivorship is debatable [43], further experiments conducted with wild P. falciparum isolates combined with enumeration of mosquito mortality prior to ivermectin ingestion are necessary. A reduced susceptibility of $P$. falciparum-infectious An. gambiae to ivermectin combined with the fact that Plasmodium sporozoite salivary gland infection alters mosquito behaviour would enhance the efficacy of ivermectin MDA for Plasmodium transmission control. Plasmodium-infectious vectors in the laboratory $[44,45]$ and the field [46,47] have increased mosquito host probing and blood feed more frequently compared to uninfected mosquitoes. In Tanzania, wild P. falciparum- 
infectious An. gambiae s.l. were more likely to feed on multiple people per night and feed to repletion compared to uninfected counterparts [47]. Multiple ivermectin-containing blood meals were found to compound An. gambiae s.s. mortality at a greater rate than a single ivermectin-containing blood meal [4]. As roughly $80 \%$ of people in a village are treated with ivermectin during routine MDAs, it follows that the more frequent host feeding of $P$. falciparum-infectious An. gambiae s.s. means that they are more likely to imbibe a blood meal that contains ivermectin or multiple blood meals that contain ivermectin. These findings may further explain some of the reduction in the proportion of $P$. falciparum-infectious An. gambiae s.s. post-ivermectin MDA [6], especially if $P$. falciparuminfectious An. gambiae are more likely to die than their uninfected counterparts.

Currently, it is not known how much ivermectin is imbibed by a mosquito during blood feeding on an orally treated human. Human pharmacokinetic data for oral ivermectin treatment is based on venous blood samples [30], however ivermectin is found in fat and dermal tissue at two to three times greater concentrations than venous plasma [48]. As mosquitoes blood feed from subdermal capillaries, the amount of ivermectin imbibed may differ from the amount present in venous plasma. This makes it difficult to predict how long laboratorydemonstrated mosquito sub-lethal sporontocidal effect of ivermectin may impact $P$. falciparum transmission dynamics in the field following ivermectin MDA. Concurrent studies are attempting to quantify the concentration of ivermectin imbibed by a mosquito compared to venous and capillary blood concentrations.

This study clearly demonstrates that ivermectin can impact P. falciparum sporogony in An. gambiae which is a critical factor to reduce Plasmodium transmission via ivermectin MDA. Since ivermectin MDA has only been demonstrated to reduce vector transmission, it may be necessary to combine ivermectin MDA with antimalarial drug treatment to clear parasites from the human population. One disadvantage of mass screening and treatment (MSAT) with anti-malarial drugs is that parasites may be transmitted between the screening and treatment interval [49]. Ivermectin MDA may address this MSAT shortcoming if ivermectin is delivered at the point of sample collection to limit Plasmodium transmission between sample processing and anti-malarial drug treatment, however, the safety and efficacy of the co-administration of ivermectin and anti-malarial drugs remains to be validated. Plasmodium falciparum gametocyte circulation time has been shown to be reduced post artemisinin-based combination therapy (ACT) [50], however, field evidence demonstrates that $P$. falciparum transmission still occurs post-ACT treatment [51-54].
Primaquine is effective against late-stage gametocytes $[55,56]$ and has been co-administered with ACT in an effort to reduce Plasmodium transmission. However, primaquine causes haemolysis in humans with glucose6-phosphate dehydrogenase deficiency $[57,58]$ which could reduce human compliance with the treatment regimen. As ivermectin has sporontocidal, mosquito lethal, and mosquito sub-lethal effects that prevent Plasmodium transmission, and does not cause haemolysis, it may be an ideal agent to combine with ACT.

\section{Conclusions}

Ivermectin is sporontocidal to $P$. falciparum at concentrations sub-lethal to An. gambiae and that are present in humans after oral treatment. Mosquito ingestion of ivermectin inhibited sporogony in An. gambiae when ingested before, with, or after parasite ingestion. Plasmodium falciparum-infectious An. gambiae may be slightly more susceptible to ivermectin than uninfected counterparts. As these studies were conducted with cultured $P$. falciparum (NF54) and colonized An. gambiae s.s. (G3 strain) it will be necessary to validate results with wild $P$. falciparum isolates and wild-caught An. gambiae or with other Plasmodium and Anopheles species combinations. These results further substantiate the utility of ivermectin MDA to reduce $P$. falciparum transmission.

\section{Abbreviations}

RBC: Red Blood Cell; DMSO: Dimethyl Sulfoxide; DPI: Days Post-Infection; DDPI: Dissected At Day Post-Infection; MDA: Mass Drug Administration; MSAT: Mass Screening And Treatment; ACT: Artemisnin-based Combination Therapy.

\section{Competing interests}

The authors declare that they have no competing interests.

\section{Authors' contributions}

KCK, BDF, and JHR designed research, KCK performed research, KCK analysed data, KCK, BDF, and JHR wrote and edited the paper, JHR provided reagents. All authors read and approved the final manuscript.

\section{Acknowledgements}

The authors would like to thank the Walter Reed Army Institute of Research Vector and Parasite Biology Department including Jittawadee Murphy,

Megan Dowler, Tatyana Savranskaya, Dipali Patel, Clara Brando, and Alejandra Zapata for their assistance with Plasmodium falciparum culture, raising mosquitoes, and monitoring experiments, Charles White for statistical consulting, and Michael O'Neil and Michelle Colacicco-Mayhugh for critical discussion and support. This work was supported in part by the Military Infectious Disease Research Program and BDF was supported by grant 1R01Al094349-01A1 from the National Institutes of Health-National Institute of Allergy and Infectious Diseases.

Material has been reviewed by the Walter Reed Army Institute of Research. There is no objection to its publication. The opinions or assertions contained herein are the private views of the author, and are not to be construed as official, or as reflecting true views of the Department of the Army or the Department of Defense.

\section{Author details}

${ }^{1}$ Entomology Branch, Walter Reed Army Institute of Research, 503 Robert Grant Ave, Silver Spring, MD 20910, USA. ²Department of Microbiology, Immunology and Pathology, Arthropod-borne and Infectious Diseases 
Laboratory, Colorado State University, 1692 Campus Delivery, Fort Collins, CO 80523-1692, USA.

Received: 9 October 2012 Accepted: 17 November 2012 Published: 21 November 2012

\section{References}

1. Alonso PL, Besansky NJ, Burkot TR, Collins FH, Hemingway J, James AA Lengeler C, Lindsay S, Liu Q, Lobo NF, Mnzava A, Tanner M, Zwiebel L: A research agenda for malaria eradication: Vector control. PLOS Med 2011, 8:e1000401.

2. Wilson ML: Avermectins in arthropod vector management - prospects and pitfalls. Parasitol Today 1993, 9:83-87.

3. Bockarie MJ, Hii JL, Alexander ND, Bockarie F, Dagoro H, Kazura JW, Alpers MP: Mass treatment with ivermectin for filariasis control in Papua New Guinea: impact on mosquito survival. Med Vet Entomol 1999, 13:120-123.

4. Kobylinski KC, Deus KM, Butters MP, Hongyu T, Gray M, da Silva IM, Sylla M, Foy BD: The effect of oral anthelmintics on the survivorship and refeeding frequency of anthropophilic mosquito disease vectors. Acta Trop 2010, 116:119-126.

5. Sylla M, Kobylinski KC, Gray M, Chapman PL, Sarr MD, Rasgon JL, Foy BD: Mass drug administration of ivermectin in south-eastern Senegal reduces the survivorship of wild-caught, blood fed malaria vectors. Malar J 2010, 9:e365

6. Kobylinski KC, Sylla M, Chapman PL, Sarr MD, Foy BD: Ivermectin mass drug administration to humans disrupts malaria parasite transmission in Senegalese villages. Am J Trop Med Hyg 2011, 85:3-5.

7. Foy BD, Kobylinski KC, da Silva IM, Rasgon JL, Sylla M: Endectocides for malaria control. Trends Parasitol 2011, 27:423-428.

8. Foley DH, Bryan JH, Lawrence GW: The potential of ivermectin to control the malaria vector Anopheles farauti. Trans R Soc Trop Med Hyg 2000, 94:625-628.

9. Cully DF, Vassilatis DK, Liu KK, Paress PS, Van der Ploeg LHT, Schaeffer JM, Arena JP: Cloning of an avermectin-sensitive glutamate-gated chloride channel from Caenorhabditis elegans. Nature 1994, 371:707-711.

10. Kane NS, Hirschberg B, Qian S, Hunt D, Thomas B, Brochu R, Ludmerer SW, Zheng YC, Smith M, Arena JP, Cohen CJ, Schmatz D, Warmke J, Cully DF: Drug-resistant Drosophila indicate glutamate-gated chloride channels are targets for the antiparasitics nodulisporic acid and ivermectin. Proc Natl Acad Sci U S A 2000, 97:13949-13954.

11. Hemingway J, Ranson H: Chemical control of vectors and mechanisms of resistance. In Biology of disease vectors. 2nd edition. Edited by Marquardt WC. San Diego: Elsevier Academic Press; 2005:627-647

12. Garrett-Jones C: Prognosis for interruption of malaria transmission through assessment of the mosquito's vectorial capacity. Nature 1964, 204:1173-1175.

13. Enayati A, Hemingway J: Malaria management: Past, present, and future. Annu Rev Entomol 2010, 55:569-591.

14. Curtis CF, Jana-Kara B, Maxwell CA: Insecticide treated nets: impact on vector populations and relevance of initial intensity of transmission and pyrethroid resistance. J Vector Borne Dis 2003, 40:1-8.

15. Black WCI, Moore CG: Population biology as a tool to study vector-borne diseases. In Biology of disease vectors. Second editionth edition. Edited by Marquardt WC. San Diego, CA: Elsevier Academic Press; 2005:187-206.

16. Fritz ML, Siegert PY, Walker ED, Bayoh MN, Vulule JR, Miller JR: Toxicity of bloodmeals from ivermectin-treated cattle to Anopheles gambiae s.l. Ann Trop Med Parasitol 2009, 103:539-547.

17. Chaccour C, Lines J, Whitty CJM: Effect of ivermectin on Anopheles gambiae mosquitoes fed on humans: The potential of oral insecticides in malaria control. J Infect Dis 2010, 202:113-116.

18. Kiszewski A, Mellinger A, Spielman A, Malaney P, Sachs SE, Sachs J: A global index representing the stability of malaria transmission. Am J Trop Med Hyg 2004, 70:486-498.

19. Butters MP, Kobylinski KC, Deus KM, da Silva IM, Gray M, Sylla M, Foy BD: Comparative evaluation of systemic drugs for their effects against Anopheles gambiae. Acta Trop 2012, 121:34-43.

20. Molyneux DH, Bradley M, Hoerauf A, Kyelem D, Taylor MJ: Mass drug treatment for lymphatic filariasis and onchocerciasis. Trends Parasitol 2003, 19:516-522.

21. Trager $W$, Jensen JB: Human malaria parasites in continuous culture. Science 1976, 193:673-675.

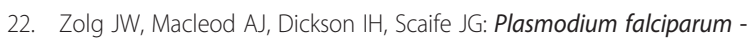
modifications of the in vitro culture conditions improving parasitic yields. J Parasitol 1982, 68:1072-1080.

23. SAS Institute I: SAS Version 9.2. Cary, NC; 2002.

24. Hibbs RE, Gouaux E: Principles of activation and permeation in an anionselective Cys-loop receptor. Nature 2011, 474:54-60.

25. Cully DF, Paress PS, Liu KK, Schaeffer JM, Arena JP: Identification of a Drosophila melanogaster glutamate-gated chloride channel sensitive to the antiparasitic agent avermectin. J Biol Chem 1996, 271:20187-20191.

26. Duce $I R$, Scott RH: GABA sensitivity in the distal bundles of the locust extensor tibiae muscle. J Physiol 1983, 343:31-32.

27. Ludmerer SW, Warren VA, Williams BS, Zheng YC, Hunt DC, Ayer MB, Wallace MA, Chaudhary AG, Egan MA, Meinke PT, Dean DC, Garcia ML, Cully DF, Smith MM: Ivermectin and nodulisporic acid receptors in Drosophila melanogaster contain both gamma-aminobutyric acid-gated Rdl and glutamate-gated $\mathrm{GluCl}$ alpha chloride channel subunits. Biochemistry 2002, 41:6548-6560.

28. Dent JA, Smith MM, Vassilatis DK, Avery L: The genetics of ivermectin resistance in Caenorhabditis elegans. Proc Natl Acad Sci U S A 2000, 97:2674-2679.

29. Nasveld P, Russell B, Kotecka B, Rieckmann K: Lack of in vitro effect of ivermectin on Plasmodium falciparum. Southeast Asian J Trop Med Public Health 2003, 34:552-553.

30. Elkassaby MH: Ivermectin uptake and distribution in the plasma and tissue of Sudanese and Mexican patients infected with Onchocerca volvulus. Trop Med Parasitol 1991, 42:79-81.

31. Lariviere M, Beauvais B, Aziz M, Garin YJF, Abeloos J, Derouin F, Bamba M, Bosseboeuf C, Ferlytherizol M, Sarfati C, Basset D, Basset A, Toure Y, Song D, Gaxotte P: Study in Ivory Coast (1985-1987) of the efficacy and tolerance of ivermectin (Mectizan) in human onchocerciasis. 1. a double-blind comparative study of 200 patients treated with a single oral dose of 100 $\mathrm{mcg} / \mathrm{kg}, 150 \mathrm{mcg} / \mathrm{kg}$ or $200 \mathrm{mcg} / \mathrm{kg}$. Bull Soc Pathol Exot 1989, 82:35-47.

32. Burg RW, Stapley EO: Isolation and characterization of the producing organism. In Ivermectin and abamectin. Edited by Campbell WC. New York, New York: Springer; 1989:24-32.

33. Mastrangelo E, Pezzullo M, De Burghgraeve T, Kaptein S, Pastorino B, Dallmeier K, de Lamballerie X, Neyts J, Hanson AM, Frick DN, Bolognesi M, Milani M: Ivermectin is a potent inhibitor of flavivirus replication specifically targeting NS3 helicase activity: new prospects for an old drug. J Antimicrob Chemother 2012, 67:1884-1894.

34. Wagstaff KM, Sivakumaran $H$, Heaton SM, Harrich D, Jans DA: Ivermectin is a specific inhibitor of importin alpha/beta-mediated nuclear import able to inhibit replication of HIV-1 and dengue virus. Biochem J 2012, 443:851-856.

35. Mahmood F, Walters LL, Guzman H, Tesh RB: Effect of ivermectin on the ovarian development of Aedes aegypti (Diptera: Culicidae). J Med Entomol 1991, 28:701-707.

36. Shahabuddin M, Toyoshima T, Aikawa M, Kaslow DC: Transmissionblocking activity of a chitinase inhibitor and activation of malarial parasite chitinase by mosquito protease. Proc Natl Acad Sci U S A 1993, 90:4266-4270

37. Shahabuddin M, Kaslow DC: Plasmodium: parasite chitinase and its role in malaria transmisison. Exp Parasitol 1994, 79:85-88.

38. Smith RC, Jacobs-Lorena M: Plasmodium-mosquito interactions: A tale of roadblocks and detours. In Advances in Insect Physiology, Vol 39. Volume 39. Edited by Simpson SJ. London: Academic Press Ltd-Elsevier Science Ltd; 2010:119-149.

39. Robert V, Le Goff G, Gouagna LC, Sinden M, Kieboom J, Kroneman R, Verhave JP: Kinetics and efficiency of Plasmodium falciparum development in the midguts of Anopheles gambiae, An. funestus and An. nili. Ann Trop Med Parasitol 1998, 92:115-118.

40. Chege GMM, Beier JC: Immunodetection of Plasmodium falciparum zygotes and ookinetes in Anopheles blood meals. J Am Mosa Control Assoc 1994, 10:419-422.

41. Meis JFGM, Wismans PG, Jap PH, Lensen AH, Ponnudurai T: A scanning electron microscopic study of the sporogonic development of Plasmodium falciparum in Anopheles stephensi. Acta Trop 1992, 50:227-236.

42. Vaughan JA, Noden BH, Beier JC: Population dynamics of Plasmodium falciparum sporogony in laboratory-infected Anopheles gambiae. J Parasitol 1992, 78:716-724. 
43. Ferguson HM, Read AF: Why is the effect of malaria parasites on mosquito survival still unresolved? Trends Parasitol 2002, 18:256-261.

44. Rossignol PA, Ribeiro JMC, Spielman A: Increased intradermal probing time in sporozoite-infected mosquitoes. Am J Trop Med Hyg 1984, 33:17-20.

45. Wekesa JW, Copeland RS, Mwangi RW: Effect of Plasmodium falciparum on blood feeding behavior of naturally infected Anopheles mosquitoes in Western Kenya. Am J Trop Med Hyg 1992, 47:484-488.

46. Koella JC, Packer MJ: Malaria parasites enhance blood-feeding of their naturally infected vector Anopheles punctulatus. Parasitol 1996, 113:105-109.

47. Koella JC, Sorensen FL, Anderson RA: The malaria parasite, Plasmodium falciparum, increases the frequency of multiple feeding of its mosquito vector, Anopheles gambiae. Proc R Soc Lond Ser B-Biol Sci 1998, 265:763-768.

48. Baraka OZ, Mahmoud BM, Marschke CK, Geary TG, Homeida MMA, Williams JF: Ivermectin distribution in the plasma and tissues of patients infected with Onchocerca volvulus. Eur I Clin Pharmacol 1996, 50:407-410.

49. Bousema T, Drakeley C: Epidemiology and infectivity of Plasmodium falciparum and Plasmodium vivax gametocytes in relation to malaria control and elimination. Clin Microbiol Rev 2011, 24:377-410.

50. Bousema T, Okell L, Shekalaghe S, Griffin JT, Omar S, Sawa P, Sutherland C, Sauerwein R, Ghani AC, Drakeley C: Revisiting the circulation time of Plasmodium falciparum gametocytes: molecular detection methods to estimate the duration of gametocyte carriage and the effect of gametocytocidal drugs. Malar J 2010, 9:e136.

51. Bousema JT, Schneider P, Gouagna LC, Drakeley CJ, Tostmann A, Houben R, Githure Jl, Ord R, Sutherland CJ, Omar SA, Sauerwein RW: Moderate effect of artemisinin-based combination therapy on transmission of Plasmodium falciparum. J Infect Dis 2006, 193:1151-1159.

52. Targett G, Drakeley C, Jawara M, von Seidlein L, Coleman R, Deen J, Pinder M, Doherty T, Sutherland C, Walraven G, Milligan P: Artesunate reduces but does not prevent posttreatment transmission of Plasmodium falciparum to Anopheles gambiae. J Infect Dis 2001, 183:1254-1259.

53. Sutherland CJ, Ord R, Dunyo S, Jawara M, Drakeley CJ, Alexander N, Coleman R, Pinder M, Walraven G, Targett GAT: Reduction of malaria transmission to Anopheles mosquitoes with a six-dose regimen of co-artemether. PLOS Med 2005, 2:338-346.

54. Okell LC, Drakeley CJ, Ghani AC, Bousema T, Sutherland CJ: Reduction of transmission from malaria patients by artemisinin combination therapies: a pooled analysis of six randomized trials. Malar J 2008, 7:e125.

55. Shekalaghe S, Drakeley C, Gosling R, Ndaro A, van Meegeren M, Enevold A, Alifrangis M, Mosha F, Sauerwein R, Bousema T: Primaquine clears submicroscopic Plasmodium falciparum gametocytes that persist after treatment with sulphadoxine-pyrimethamine and artesunate. PLOS Clin Trials 2007, 2:e1023.

56. Pukrittayakamee S, Chotivanich K, Chantra A, Clemens R, Looareesuwan S, White NJ: Activities of artesunate and primaquine against asexual- and sexual-stage parasites in falciparum malaria. Antimicrob Agents Chemother 2004, 48:1329-1334

57. Reeve PA, Toaliu H, Kaneko A, Hall JJ, Ganczakowski M: Acute intravascular hemolysis in Vanuatu following a single dose of primaquine in individuals with glucose-6-phosphate-dehydrogenase deficiency. J Trop Med Hyg 1992, 95:349-351.

58. Brewer GJ, Zarafonetis CJD: Haemolytic effect of various regimens of primaquine with chloroquine in American negroes with G6PD deficiency and lack of an effect of various antimalarial suppressive agents on erythrocyte metabolism. Bull World Health Organ 1967, 36:303-308.

doi:10.1186/1475-2875-11-381

Cite this article as: Kobylinski et al: Ivermectin inhibits the sporogony of Plasmodium falciparum in Anopheles gambiae. Malaria Journal 2012 $11: 381$

\section{Submit your next manuscript to BioMed Central and take full advantage of:}

- Convenient online submission

- Thorough peer review

- No space constraints or color figure charges

- Immediate publication on acceptance

- Inclusion in PubMed, CAS, Scopus and Google Scholar

- Research which is freely available for redistribution 